\title{
Image 2:027 Radiologic-Pathologic Correlation: Primary Breast Lymphoma
}
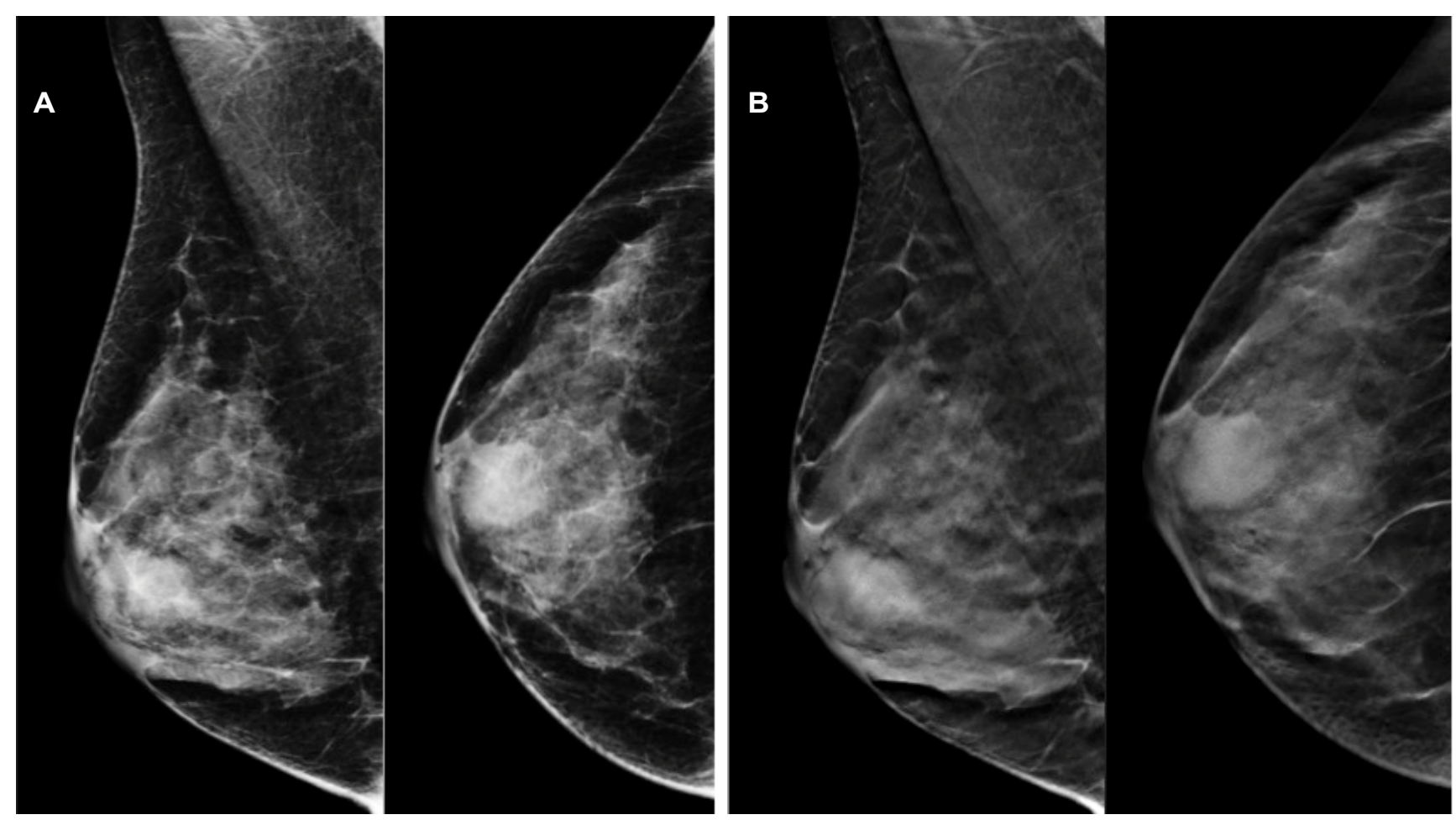

Figure 1: Mammography (a) and tomosynthesis (b) showing oval mass, with circumscribed margins, with no evidence of fat component.

A 59-year-old woman presented with a 1-week history of a palpable lump in the right breast. She denied any other symptoms and was otherwise in good health, without any significant findings in the medical or surgical history. There was no family or personal history of breast cancer.

At physical examination, the markedly asymmetric right breast was filled with a soft mass in the intersection of the inferior quadrants. There were no skin changes, nipple discharge, or lymphadenopathy. The left breast appeared normal and small in size.

Further evaluation included mammography and ultrasonography (US). Mammography demonstrated a $2.5 \mathrm{~cm}$ oval mass, with circumscribed margins. US showed a solid mass, oval and circumscribed. The eco pattern was heterogeneous, showing hypo and hyperechoic areas, suggesting fat containing lesion. No vascularity was found with Doppler. Elastography demonstrated soft stiffness. Posteriorly, tomosynthesis was performed and no fat was found in the lesion. In face of these findings, core biopsy was executed and demonstrated diffuse large B-cell lymphoma (Figure 1 and Figure 2).

\section{Information}

\section{Bernardo Procaci Kestelman*}

Faculdade de Ciências Médicas da Santa Casa de São Paulo, Brazil

*Correspondence: Bernardo Procaci Kestelman, Medical Student at Faculdade de Ciências Médicas da Santa Casa de São Paulo, Victor Rebelo Procaci, Medical residente at Universidade Federal de Sao Paulo, Fabiola Procaci Kestelman, Medical radiologist at Hospital Federal do Andarai and Clinica Cavallieri, Brazil,Email: fabkest@gmail.com; bekest@hotmail.com
Citation: Kestelman BP (2016) Radiologic-Pathologic Correlation: Primary Breast Lymphoma. Clin Med Img Lib 2:027

Published: February 24, 2016

Copyright: () 2016 Kestelman BP. This is an open-access content distributed under the terms of the Creative Commons Attribution License, which permits unrestricted use, distribution, and reproduction in any medium, provided the original author and source are credited. 


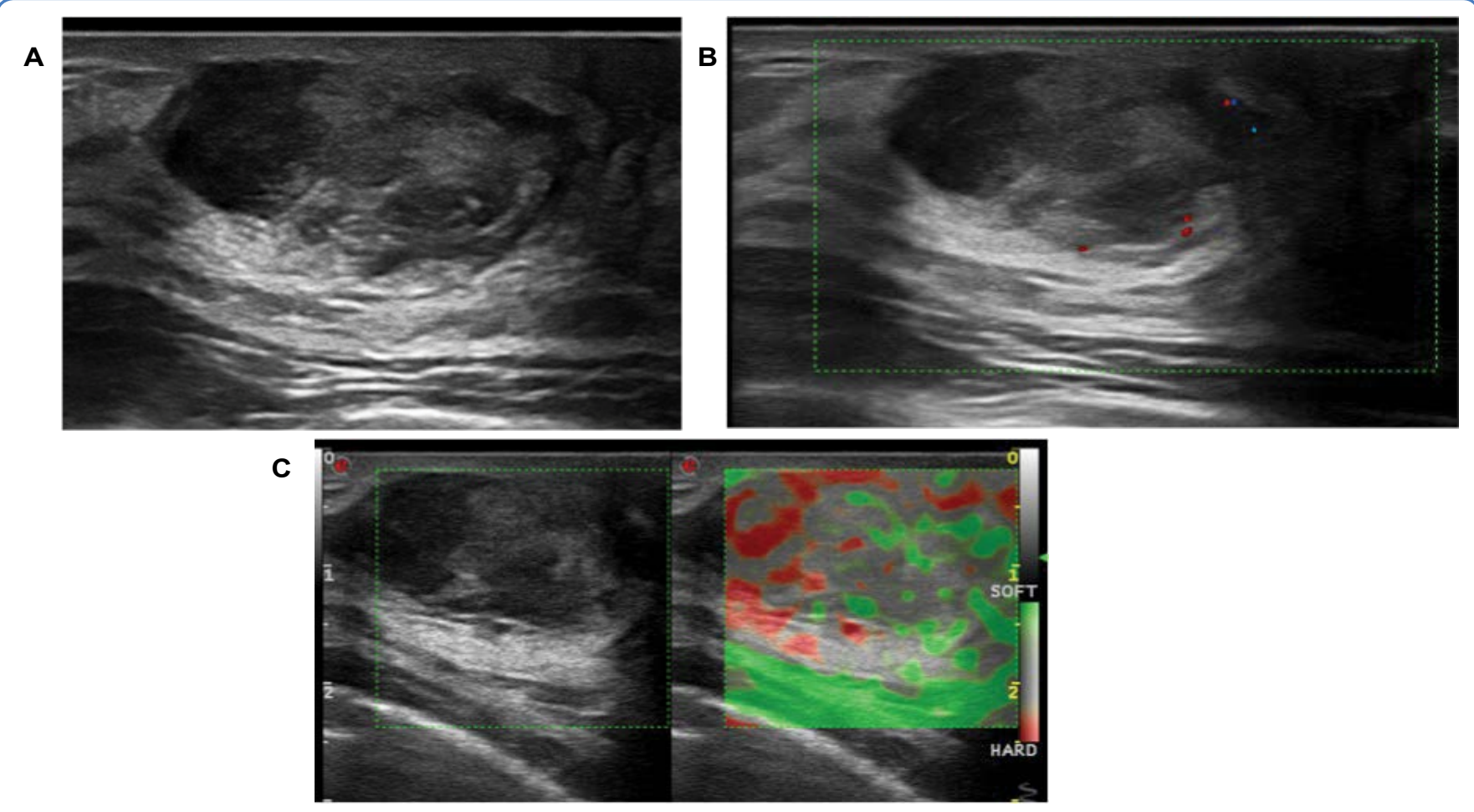

Figure 2: B mode US demonstrates (a) solid mass, oval and circumscribed, heterogeneous, with hypo and hyperechoic areas, suggesting fat containing lesion. Doppler (b) showed no flow and elastography (c) demonstrated soft stiffness.
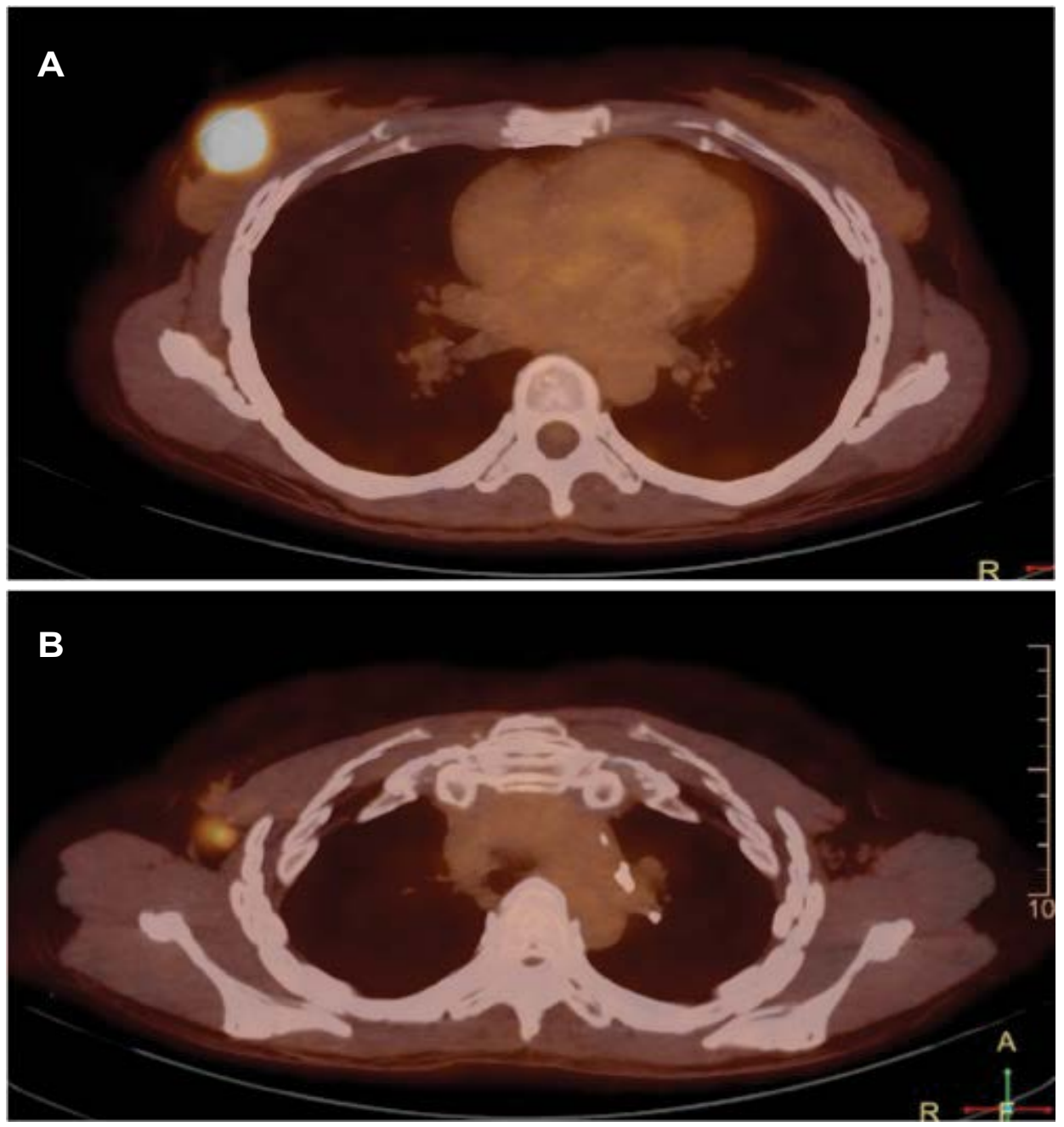

Figure 3: 18F-FDG PET/CT evidencing two images with increase FDG uptake. The abnormality in the breast was suggested to be the primary lesion (a) and only one lymph node was found in the ipsilateral axilla (b). 
A PET-CT was performed to evaluate the stage of the disease and showed two images with increased FDG uptake, corresponding to the primary lesion in the breast and one lymph node in ipsilateral axila (Figure 3a and Figure 3b).

Primary breast lymphoma (PBL) is a rare disease accounting for $0.4-0.5 \%$ of all breast malignancies. Diffuse large B-cell lymphoma is the most common histological diagnosis. The clinical presentation of PBL is usually no different from that of carcinoma. A painless mass is the most common presenting sign in PBL occurring in approximately $61 \%$ of cases [1].

Intramammary masses, most of which are round or oval in shape with circumscribed or gently lobulated margins, are the most common mammographic findings. Sonographically, breast lymphoma appears as a hypoechoic to almost anechoic round or oval-shaped mass with relatively well-defined margins with or without posterior acoustic enhancement [2].

In our case, the first evaluation with mammography and US pointed to a benign disease, however the recent manifestation of palpable lump made imperative the histopathological evaluation. The complete investigation led us to an accurate diagnosis and made possible the treatment with chemotherapy, which provided complete radiologic response.

\section{References}

1. Joks M, Mysliwiec K, Lewandowski K (2011) Primary breast lymphoma - a review of the literature and report of three cases. Arch Med Sci 7: 27-33.

2. Ha KY, Wang JC, Gill JI (2013) Lymphoma in the breast. Proc (Bayl Univ Med Cent) 26: 146-148. 